\title{
Grégoire le Grand, Le Pastoralet, Traduction médiévale française de la Regula pastoralis
}

\section{Barbara Ferrari}

\section{(2) OpenEdition}

1 Journals

\section{Edizione digitale}

URL: http://journals.openedition.org/studifrancesi/6196

DOI: ERREUR PDO dans /localdata/www-bin/Core/Core/Db/Db.class.php L.34 : SQLSTATE[HYO00]

[2006] MySQL server has gone away

ISSN: 2421-5856

\section{Editore}

Rosenberg \& Sellier

\section{Edizione cartacea}

Data di pubblicazione: 1 novembre 2010

Paginazione: 528-529

ISSN: 0039-2944

\section{Notizia bibliografica digitale}

Barbara Ferrari, «Grégoire le Grand, Le Pastoralet, Traduction médiévale française de la Regula pastoralis», Studi Francesi [Online], 162 (LIV | III) | 2010, online dal 30 novembre 2015, consultato il 08 janvier 2021. URL: http://journals.openedition.org/studifrancesi/6196; DOI: https://doi.org/ERREUR PDO dans /localdata/www-bin/Core/Core/Db/Db.class.php L.34 : SQLSTATE[HY000] [2006] MySQL server has gone away

Questo documento è stato generato automaticamente il 8 janvier 2021.

\section{(c)}

Studi Francesi è distribuita con Licenza Creative Commons Attribuzione - Non commerciale - Non opere derivate 4.0 Internazionale. 


\title{
Grégoire le Grand, Le Pastoralet, Traduction médiévale française de la Regula pastoralis
}

\author{
Barbara Ferrari
}

\section{NOTIZIA}

GRÉGOIRE LE GRAND, Le Pastoralet. Traduction médiévale française de la Regula pastoralis.

Édition critique du manuscrit 868 de la Bibliothèque municipale de Lyon. Édition en visà-vis du manuscrit Cotton Vitellius F VII de la British Library de Londres, publié par Martine Pagan, Paris, H. Champion, 2007 («Les Classiques français du Moyen Âge», 154), pp. 584.

1 La Regula Pastoralis di Gregorio Magno, destinata in origine ai vescovi italiani, conobbe fin dal periodo immediatamente successivo alla sua composizione (fine del vi sec.), e per tutto il Medioevo, una vastissima diffusione (500 manoscritti latini censiti, almeno 10 incunaboli); la sua popolarità è attestata inoltre dai numerosi volgarizzamenti in inglese, francese, italiano e castigliano. Le quattro parti che compongono l'opera hanno avuto anche una trasmissione indipendente, come attesta il Pastoralet, redatto in Francia all'inizio del secolo XIII, che costituisce appunto la traduzione della Tertia pars. In essa, Gregorio illustra come il pastore debba adattare il proprio insegnamento alle diverse categorie di fedeli, considerate via via in coppie antitetiche (donne/uomini, giovani/vecchi, ricchi/poveri, ecc.), offrendo al lettore, oltre a una lezione di metodo, «un ouvrage susceptible de le mener, par la connaissance de l'autre, jusqu'à l'autoanalyse» (p. 15).

2 Martine Pagan fornisce l'edizione di due diverse redazioni del testo francese corredata da numerosi apparati e preceduta da un'importante Introduzione articolata in quattro sezioni. La prima è dedicata alla presentazione del trattato latino e della sua fortuna nel Medioevo («La Regula pastoralis», pp.11-25), mentre la seconda affronta la tradizione 
testuale del volgarizzamento attraverso l'esame dei testimoni e la loro classificazione («Le Pastoralet», pp. 25-103). Trasmesso da 7 mss. copiati tra il XIII e il xV secolo, il Patoralet ci è pervenuto all'interno di sillogi di testi religiosi, in cinque casi su sette leggendari; la parentela tra le diverse raccolte ha costituito quindi il primo criterio per stabilire i rapporti di filiazione tra i testimoni, a questo si aggiungono la datazione, l'analisi dei tratti linguistici e, naturalmente, la collazione completa delle lacune e delle varianti (senza tuttavia isolare e discutere gli errori significativi). L'antichità (terzo quarto del XIII sec.) e la fedeltà al testo latino, sono le ragioni principali che inducono $\mathrm{M}$. Pagan a scegliere come manoscritto base A (Lyon, BM, 868, ant. 773), relatore della cosiddetta versione 'champenoise' delle Vitae Patrum, eseguita, secondo P. Meyer, tra la fine del XII secolo e il primo quarto del XIII per Bianca di Navarra, sposa o forse già vedova di Thibaut III di Champagne (HLF, XXXIII, pp. 292-312); secondo l'editrice, anche per il Pastoralet (il cui testo segue quello delle Vitae Patrum) si potrebbe ipotizzare la stessa committenza. L'analisi della lingua del manoscritto (grafia, fonetica, morfologia; pp. 81-96) individua un'area di provenienza abbastanza vasta che comprende tutta la parte orientale del dominio d'oil. M. Pagan sceglie inoltre di dare a fronte il testo anglonormanno di $G$ (London, BL, Cotton Vitellius F VII), copia diretta del ms. base, come mostra tra l'altro l'integrazione a testo delle correzioni e delle note marginali inserite dal revisore di $A$. La decisione insolita di editare anche il testo di un descriptus è giustificata dall'interesse linguistico di questo testimone: «En éditant le manuscrit $G$, nous avons souhaité rendre compte d'un processus de copie par un scripteur qui ne traduit pas une langue étrangère dans la sienne propre, mais qui ne copie pas, non plus, un texte dans une langue telle qu'il la pratique» (p. 102).

3 Nella terza parte dell'Introduzione, la più ampia («étude des 'transferts linguistiques'», pp.103-217), M. Pagan analizza il volgarizzamento prendendo in considerazione dapprima le operazioni linguistiche à livello 'intra-linguale' (segmentazione e interpunzione del testo di $A$, lavoro del revisore, procedimenti di riscrittura nella copia tardiva $D, B n F$, fr. 24864, e in quella anglonormanna $G$ ), e studiando in seguito, in una prospettiva verticale 'interlinguale', il trattamento riservato dal traduttore al testo latino (riorganizzazione della materia in funzione dei nuovi destinatari, scelte traduttorie a livello di significante e di significato). Raramente nell'introduzione di un'edizione critica trova spazio un'analisi così approfondita, di cui, nell'impossibilità di darne conto compiutamente, va sottolineato l'interesse e la ricchezza di spunti.

4 La quarta e ultima sezione dell'Introduzione è costituita da un'ampia Bibliografia (pp. 217-39); segue l'edizione vera e propria (pp. 241-443). Il testo di A, post correctionem, è riprodotto fedelmente; i rari interventi necessari, trattandosi di un testo già sottoposto a revisione, sono chiaramente indicati nell'apparato a pié di pagina, mentre le varianti, associate alle note, sono collocate in fondo (pp. 445-503). A fronte, come si è detto, è editato il testo di $G$. Purtroppo l'accuratezza della trascrizione lascia talvolta a desiderare, come segnala, riportando alcuni esempi, anche F. Duval nella sua recensione sulla Bibliothèque de l'École des Chartes (166, 2008, pp. 243-45: disomogeneità nell'uso delle maiuscole e nella separazione delle parole, punteggiatura non sempre adattata all'uso moderno, come dichiarato nell'introduzione); ciò che però disturba la lettura è un ricorrente errore tipografico per cui al termine del discorso diretto sono stampate spessissimo virgolette di apertura anziché di chiusura (un solo esempio: ... qu'il ne dit mie: «Prie «mas: «Commande, «et ce dit il ..., p. 243). Senz'altro non imputabile all'editrice è la discutibile mise en page che lascia margini inferiori spesso superiori ai 5 
$\mathrm{cm}$, dove avrebbero ampiamente e utilmente trovato posto le varianti confinate in fondo; inoltre, inspiegabilmente, solo il testo di $G$ è giustificato, mentre quello di $A$ è allineato a sinistra.

5 L'edizione è arricchita da una tavola dei paralleli testuali tra i Moralia in Job in vallone (ed. Foerster 1965) e il Pastoralet (pp. 505-14); dall'elenco dei rinvii ai passi biblici (pp. 515-25) e dall'indice dei nomi propri (pp. 527-31). L'ultima parte del volume (pp. 533-48) è riservata al «Lexique», che l'editrice dichiara di aver preferito ad un glossario di fattura tradizionale, senza tuttavia specificare le caratteristiche che differenzierebbero l'uno dall'altro. La scelta dei termini tiene conto dei temi principali che percorrono l'opera e delle possibili difficoltà di comprensione. Da apprezzare la presenza, ove possibile, della parola o del sintagma latino all'origine del termine francese. 